\title{
Research on Construction of Collaborative Innovation System for Realization of More Attractive Higher Vocational Education*
}

\author{
Jie Liu \\ Urumqi Vocational University \\ Urumqi, China
}

\begin{abstract}
Construction and improvement of collaborative innovation system are of great importance in constructing education model in many ways, promoting talent training and strengthening the attraction of higher vocational education. According to problems in construction of collaborative innovation system in higher vocational education, suggestions and opinions on the construction of policy system related to collaborative innovation, information sharing mechanism, benefit sharing mechanism and collaborative education mechanism are proposed, in order to provide references for the improvement of depth and integration of cooperation between school and enterprise, education quality and social attraction of higher vocational education.
\end{abstract}

Keywords-higher vocational education; attraction; collaborative innovation; mechanism

\section{INTRODUCTION}

Huang Yanpei proposes the purpose of vocational education is "make jobless person employed and make employees have professional enthusiasm" and thinks education must develop personality, the sound development of which depends on good vocational education, in order to train people's qualities required by social and economic life. The training objective of higher vocational education in China is: train senior technical application talents who meet the demand of Socialist Modernization, grasp essential theories and professional knowledge and have comprehensive qualities and vocational abilities and work in front line. One of the "comprehensive qualities" is creative spirit and innovation ability. The training of innovation ability is the basic difference between modern education and traditional education. Innovation ability is intellectual characteristic, personality characteristic and positive mental state. Higher vocational education aims at training senior skilled talents who serve at the front line and lays foundation for modern social and economic development. Although attentions are paid to vocational education that has developed rapidly, compared

*Project 1: 2010 Key topic of research on development strategy of vocational education of the Chinese Society of Vocational and Technical Education (No.: ZLZ04): Research on Strengthening Social Attraction of Higher Vocational Colleges

*Project 2: 2016 school level key project of World Bank loan of Urumqi Vocational University (Project No.: 2016XZ002): Research on Development Strategy of Urumqi Vocational Education Group. with traditional academic education, vocational education is less popular in society. After college entrance examination, students prefer regular institutions of higher learning to higher vocational colleges. Some are reluctant to choose higher vocational colleges through adjusting their majors. Majority of employers prefer to students graduated from universities of "985 Project" and "211 Project" in employment. In recent years, the increasingly serious "structural labor shortage" also indicates higher vocational education is less attractive: In Zhejiang, Hangzhou Confederation of Trade Unions investigates hundred of enterprises in construction industry and traditional manufacturing industry in Hangzhou in 2016. The results show 71.43 percent of enterprises lack senior skilled workers and have surplus low skilled labors. ${ }^{1}$ Colleges students fail to find jobs suited to their professional training. Enterprises cannot recruit senior skilled talents. It is caused by the prejudice towards higher vocational colleges, which will influence students' employment and restrict economic development and to which attentions should be paid. The Outline of the National Program for Medium and Long Term Educational Reform and Development (2010-2020) also emphasizes the importance to strengthen attraction of vocational education.

II. SigNIFICANCE OF CONSTRUCTING COLLABORATIVE INNOVATION MECHANISM ON SOCIAL ATTRACTION OF HIGHER VOCATIONAL EDUCATION

Except for social prejudice, education quality and curriculum provision of higher vocational education cannot keep abreast with social and economic development, leading to less attractive higher vocational education, which embodies in the structural "labor shortage". Higher vocational colleges lack "double-professionally-titled teachers" with theories and skills. Teaching contents fail to meet demands of industry, enterprises and society for talents, leading to low teaching quality. In order to improve the level of "double-professionally-titled teachers" in higher vocational colleges and make curriculum provision more reasonable to meet demands of social and economic development, it is not enough to depend on policies of teaching administrative departments and results of research

1 Shang Yiying. Jobless Growth Appearing in the East-Employment Structure Enters Transformation Period [N], Economic Daily, the $5^{\text {th }}$ Edition on April 12, 2013 
institutions of vocational education. Schools, enterprises and government sectors must carry out collaborative innovation and reform the talent training model of higher vocational education, in order to make higher vocational education more attractive.

On April 24, 2011 on centennial anniversary of Tsinghua University, $\mathrm{Hu}$ Jintao clearly proposed "actively promote collaborative innovation and encourage universities to cooperate with scientific research institutions and enterprises through mechanism innovation and policy guidance, establish strategic alliances of innovative innovation and promote resource sharing to make contributions for innovation-oriented country". In April 2014, Ministry of Education and Ministry of Finance jointly issued 2011 Construction and Development Plan for Collaborative Innovation Center, 2011 Opinions on Policy Support of Collaborative Innovation Center, 2011 Interim Measures for Identification of Collaborative Innovation Center, proposing "according to the general requirement of national need, world first class, advanced system and prominent contribution, give full play to the unique role of higher education as combination of No.1 productive force and human resources, gather excellent innovation team, resources and innovate in talent training model driven by collaborative innovation tasks and guaranteed by mechanism reform to meet social demands". Under the guidance of "2011 Plan", some "collaborative innovation centers" have been constructed. Provinces, autonomous regions and municipality directly under the central government introduce plans of collaborative innovation, indicating collaborative innovation is becoming profound. High attentions have been paid to collaborative innovation development of higher vocational education and social force. However, the benign development depends on sound frame of collaborative innovation system and operation mechanism. Construction and improvement of collaborative innovation mechanism of higher vocational education avails the improvement of attraction of vocational education.

\section{KEY PROBLEMS IN COLLABORATIVE INNOVATION SySTEM OF VocATiONAL EdUCATION AT THE PRESENT STAGE}

In recent years, vocational education develops rapidly and has made achievements in talent training quality and connotation construction. However, it lacks mature experience and mechanism in cooperative education and innovation. The unsound mechanism of collaborative innovation and the lack of systematic operation mechanism and collaboration experience will restrict continuous benign development of vocational education and education through cooperation of school, enterprise, industry and research.

\section{A. Unsound Collaborative Management and Incentive Mechanism, Ambiguous Rights and Liabilities and Interests and Different Attitudes toward Anticipated Profits Influence the Collaborative Enthusiasm}

Initially, subjects of collaborative innovation including vocational colleges, regular universities, scientific research institutions, government and scientific and technological enterprises fail to understand nature and values of innovation resources such as technology, knowledge, talents, equipment and information. Therefore, they neither excavate, make the most of resources and nor judge and find future values (potential values of resources) of resources. Collaborative innovation organizations are popular because it covers the shortage of these subjects and gives full play to the potential values of resources. [1] Furthermore, insufficient recognition makes subjects fail to estimate residual interests produced by follow-up collaborative innovation when drafting organizational structure and letter of intent or contract related to collaboration. Ambiguous ownership and distribution of industry, rights, liabilities and interests will influence the distribution of follow-up residual interests and then the enthusiasm of members in innovation.

\section{B. Government Fails to Play A Role in Collaborative}

Innovation of School, Enterprise, Industry and Research; the Lack of Operation Mechanism of Law Disenables Collaborative Innovation Mechanism to Operate Benignly

As a member or guider of collaborative innovation organization, government formulates policies and institutions as well as legal system to make collaborative innovation organizations develop and achieve results. Cooperation of industry, university and research in collaborative innovation depends on good policy support and legal system. Incomplete policies and laws will directly restrict enthusiasm of interested parties in collaboration and influence the implementation of collaborative innovation.

\section{Unreasonable Interest Distribution Mechanism or Insufficient Participation of Members Will Influence the Follow-Up Collaborative Innovation}

Interest distribution mechanism is an important mechanism of collaborative innovation organizations and requires equal and reasonable distribution of interests according to contributions made by members. It will directly influence the collaborative enthusiasm of members. [2]

\section{Unsound Resource Sharing Mechanism Restricts the Continuous and Benign Development of Collaborative Innovation Teams}

In collaborative innovation, each unit has resource superiority and unique and mature innovative platforms, other units will have difficulties in using platforms because of mechanism, thus influencing innovation activities. For example: At present, national systems only recognize the first application or completion unit instead of follow-up participants in statistics of scientific research achievements, so units regard themselves as the first completion unit and the achievements as their own. [3] Therefore, the achievements of other units created on innovative platform of this unit and of people in other units assisted by this unit have little relationship with the out of achievements of this unit, influencing the enthusiasm of this unit that provide platforms in participating in the innovative ac 


\section{E. Collaborative Innovation Lacks Talent Training Mechanism, and Attentions Are Not Paid to the Construction of Collaborative Innovation Team}

Previously, most collaborative innovation bases on projects to share out the work and cooperate. Innovative teams dissolve after the project is completed. Insufficient attentions are paid to team construction and training of young talents, failing to realize sustainable collaborative innovation.

\section{EFFECTIVE WAYS TO CONSTRUCT COLLABORATIVE INNOVATION MECHANISM OF VOCATIONAL EDUCATION AND SUGGESTIONS}

\section{A. Improve Laws and Policies Related to Collaborative Innovation}

Matched policies and systems are formulated according to the development needs of collaborative innovation. When necessary, ensure legal interests of parties in collaborative innovation through legislation. In the development of collaborative innovation organizations, external conditions such as market environment, technology and resources of collaborative subjects, laws and regulations and policies influence the realization of goals and the improvement of efficiency. Preferential tax policy for enterprises and policies of intellectual property protection of higher vocational teachers that participate in the collaborative innovation must be complete. At the present stage, the enthusiasm of traditional enterprises in participating in collaborative innovation hasn't been aroused, so government should provide preferential tax policy. The system of intellectual property rights protection guarantees smooth implementation of collaborative innovation and stimulate enthusiasm of participants who will create more innovative products.

\section{B. Establish and Improve Information Sharing Mechanism of Collaborative Innovation}

Information sharing platforms are established to make information exchange smoother and more efficient and maximize collaborative performance. With legal safeguard and system safeguard of explicit rights and liabilities, establishment and improvement of information resource platforms reduce communication costs and let participants clearly plan innovative directions and objectives and bring resource superiority into full play.

\section{Improve Interest Distribution Mechanism of Collaborative Innovation}

Furthest following the principle of input-output equality, principles of fairness and efficiency and formulating and supervising interest distribution model will avail the operation of interest distribution mechanism.

\section{Build Education Mechanism of Collaborative Innovation}

Collaborative innovation development of vocational education promotes social and economic development, but the ultimate goal is education. The construction of systematic education system is significant to realize continuous development of collaborative innovation organizations. The construction of education mechanism of collaborative innovation depends on: First, schools strengthen course reform and cooperate with experts, senior technicians and managers of enterprises, and bring the latest technologies and information about demand for talents and economic development concepts in classroom, in order to improve students' social adaptation ability. Second, the training of "double-professionally-titled teachers" must cooperate with enterprises and dispatch teachers to learn latest technology and management styles in front line of enterprises. Meanwhile, skillful craftsmen of enterprises should be invited to classroom and improve students' practical operation ability. Third, construction of campus culture is strengthened through integrating education of occupational culture, in order to train students' professional quality and ethics.

\section{CONCLUSION}

With rapid social and economic development and everchanging demands for talents, vocational education must train talents that can meet market demands, so it will be recognized by society and enterprises and meet demands of economic development, as well as improve social attraction. The construction of collaborative innovation mechanism avails the sufficient and deep collaboration and integration of industry, enterprise, school, research and government to promote development and social attraction of vocational education.

\section{REFERENCES}

[1] Shi Zhuqin, Cai Ruilin, Zhu Xianqi, Research on Collaborative Innovation Mechanism under the Perspective of Barzel's Property Rights Theory [J], Science and Technology Progress and Policy, 2017, the $4^{\text {th }}$ Issue

[2] $\mathrm{Fu}$ Junchao. Research on Industry-university-research Cooperation Mechanism and Performance Evaluation [D]: [doctoral thesis], Wuhan: School of Economics and Management of China University of Geosciences, May 2013

[3] Wang Hongtao, Yu Hongbo, Guo Linxiang, Zhang Zhenqi, Liu Enxian, Zhang Hui. Problems in Construction of Collaborative Innovation Center Mechanism of Local Colleges and Countermeasures [J], Acta Academiae Medicinae Qingdao Universitatis, 2017, the $1^{\text {st }}$ Issue. 